\title{
Dioscin facilitates ROS-induced apoptosis via the p38-MAPK/HSP27-mediated pathways in lung squamous cell carcinoma
}

\author{
Yinan Yao\#, Luyun Cui\#, Jiani Ye, Guangdie Yang, Guohua Lu, Xiaomei Fang, Zhu Zeng, Jianying Zhou ${ }^{\bowtie}$ \\ Department of Respiratory Medicine, The First Affiliated Hospital, College of Medicine, Zhejiang University, Hangzhou, China. \\ \#These authors contributed equally to this work. \\ $\triangle$ Corresponding author: Jianying Zhou, E-mail: zjyhz@zju.edu.cn; Department of Respiratory Medicine, The First Affiliated Hospital, College of Medicine, \\ Zhejiang University, Qingchun Road 79, Xiacheng District, Hangzhou, China.
}

(C) The author(s). This is an open access article distributed under the terms of the Creative Commons Attribution License (https://creativecommons.org/licenses/by/4.0/). See http://ivyspring.com/terms for full terms and conditions.

Received: 2020.03.06; Accepted: 2020.08.23; Published: 2020.09.02

\begin{abstract}
Lung squamous cell carcinoma (SCC) is one of the deadliest cancers both in China and worldwide. To date, the efficacy of lung SCC treatments is limited. Recent studies have elucidated the powerful anti-tumour role of dioscin in different human cancers. Here, our study aims to investigate the effect of dioscin on lung SCC and its underlying mechanism. First, we found that dioscin not only inhibited cell proliferation and cell migration and induced cell apoptosis in lung SCC cells but also suppressed tumour growth in tumour-bearing mice. Furthermore, we noted that the accumulation of intracellular reactive oxygen species (ROS) was triggered by dioscin in lung SCC cells, leading to the phosphorylation of HSP27 through p38-MAPK and consequent cell apoptosis. The activation of p38-MAPK/HSP27 induced by the P38-MAPK activator Anisomycin enhanced the apoptosis of lung SCC cells, while the ROS inhibitor $\mathrm{N}$-acetyl-L-cysteine (NAC) and the p38-MAPK inhibitor SB203580 both attenuated dioscin-mediated cell apoptosis. Moreover, NAC suppressed the activation of p38-MAPK/HSP27 that induced by dioscin. In conclusion, these results confirm that dioscin facilitates ROS-induced apoptosis via the p38-MAPK/HSP27-mediated pathway in lung SCC.
\end{abstract}

Key words: lung SCC, dioscin, cell apoptosis, ROS; p38-MAPK

\section{Introduction}

According to global cancer statistics, lung cancer ranks first in terms of incidence and cancer-related mortality worldwide [1, 2]. Lung squamous cell carcinoma (SCC) is tobacco-related and accounts for approximately about $20-30 \%$ of lung cancers [3, 4]. The rapid development of targeted therapy promotes precision cancer therapy [5]. However, in lung SCC, with the lack of effective targetable mutations, combination chemotherapy is still the standard therapy and has limited benefit [3]. Hence, it is essential to seek potential targets and novel therapeutic options for the treatment of lung SCC.

Recently, dioscin, a steroidal saponin isolated from the roots of Dioscorea plants, has shown promising medicinal value in regulating inflammation, immunity, lipidemic and tumour development [6]. Interestingly, previous studies also confirmed the protective effect of dioscin against doxorubicin-induced cardiotoxicity [7]. Notably, abundant evidence suggests dioscin can effectively inhibit different cancers, including lung cancer, pancreatic cancer, gastrointestinal cancer and breast cancer [6, 8-11]. Dioscin exerts its anticancer effect by inducing cell cycle arrest and regulating TIGARmediated autophagy in liver cancers [9, 10]. Moreover, dioscin has been confirmed to induce DNA damage and cell apoptosis and to inhibit cell proliferation and cell invasion in human lung cancer cells $[11,12]$. Although the application of dioscin has been assessed in several tumours, the effect of dioscin 
on lung SCC and the underlying mechanism are still unknown.

Reactive oxygen species (ROS), a sub-product of oxidative energy metabolism, is vital in regulating cell functions, such as proliferation, differentiation, migration and death [13]. Recent studies confirmed that the accumulation of intracellular ROS caused by external stimuli, including dioscin, can trigger cell apoptosis through relevant signalling pathways [13-15]. In human oesophageal cancer cells, dioscin inhibits the expression of peroxiredoxins to induce ROS-mediated cell apoptosis [15]. Moreover, dioscin upregulates ROS levels to accelerate DNA damage, mitochondrial dysfunction and subsequent cell apoptosis in other cancers $[16,17]$. Hence, we consider that the anti-tumour effect of dioscin on cell apoptosis is associated with ROS.

Intracellular ROS is also regarded as a significant modulator of the p38-MAPK signalling pathway in cell apoptosis in various cancers [14, 18-20]. For example, in lung cancer cells, the accumulation of intracellular ROS caused by paraquat activates p38-MAPK and thus modulates the mitochondrial apoptotic pathway [18]. Dioscin is reported to suppress the growth of human laryngeal cancer cells by activating p38-MAPK-mediated apoptosis [21]. Thus, we reasonably believe that dioscin-induced cell apoptosis may be related to p38-MAPK. Heat shock protein 27 (HSP27) is a downstream target of p38-MAPK and participates in proteasome-mediated protein degradation, cytoskeleton remodelling and apoptosis [22, 23]. Although some previous studies have indicated that HSP27 phosphorylation contributes to p38-MAPK-induced apoptosis, it may play a cytoprotective role under certain conditions $[24,25]$. We still need further exploration of the relationship between dioscin-mediated cell apoptosis and the p38-MAPK/HSP27 signalling pathway.

We hypothesized that dioscin could induce cell apoptosis through the ROS-mediated p38-MAPK/ HSP27 signalling pathway in lung SCC cells. To confirm our hypothesis, we evaluated the effect of dioscin on lung SCC both in vivo and in vitro and then elucidated the underlying signalling pathways.

\section{Materials and Methods}

\section{Cell lines and Reagents}

Human lung SCC cell line SK-MES-1 and human bronchial epithelial cell line HBE were bought from the Committee on Type Culture Collection of the Chinese Academy of Sciences (Shanghai, China). And NCI-H520 was a gift from Dr Ying (Department of Respiratory Diseases, Sir Run Run Shaw Hospital, Zhejiang University, Hangzhou, China). All cells were cultured in RPMI-1640 (Hyclone, Logan, UT, USA) with $10 \%$ fetal bovine serum (FBS, Gibco BRL Co., Ltd., Houston, TX, USA), $100 \mathrm{U} / \mathrm{ml}$ penicillin and 100 $\mathrm{U} / \mathrm{ml}$ streptomycin (Solarbio, Beijing, China), at $37^{\circ} \mathrm{C}$ in $5 \% \mathrm{CO}_{2}$. Dioscin with the purity of over $98 \%$ was purchased from Shanghai Tauto Biochemical Technology Co., Ltd (Shanghai, China). Dioscin was dissolved in dimethyl sulfoxide (DMSO) (Sangon Biotech, Shanghai, China) at a concentration of $20 \mathrm{mM}$ and stored at $-20^{\circ} \mathrm{C}$. The final concentration of DMSO in all treatments was less than $0.1 \%$. The p38 inhibitor SB203580 and the p38 activator Anisomycin were purchased from Selleck Chemicals (Houston, TX, USA), while reactive oxygen species inhibitor antioxidant N-Acetyl-L-cysteine (NAC) was purchased from Sigma-Aldrich (St. Louis, MO, USA).

\section{Cell viability assay}

Lung SCC cells and HBE cells were seeded in 96-well plates. Then cells were treated with different concentrations of dioscin for $24 \mathrm{~h}$ and $48 \mathrm{~h}$. After that, cell counting kit-8 (Dojindo Laboratories, Tokyo, Japan) were added into cells with 1-2 $\mathrm{h}$ incubation and the OD value was read by SpectraMax i3x MulitiMode Microplate Reader (Molecular Devices, San Francisco, CA, USA) at $450 \mathrm{~nm}$.

\section{Colony formation assay}

NCI-H520 and SK-MES-1 cells were seeded in 6-well plates at a density of 500 cells/well. After overnight adhesion, cells were treated with different concentrations of dioscin $(0,1.25,2.5,5 \mu \mathrm{M})$ for $7-10$ days. Then, cells were stained with $0.1 \%$ crystal violet and imaged.

\section{Wound healing assay}

When NCI-H520 and SK-MES-1 cells reached $80 \%$ confluency in six-well plates, the cells were wounded with a sterile $10 \mu \mathrm{l}$ pipette tip on the cell monolayers and washed with serum-free medium to remove detached cells. Next, the cells were treated with dioscin $(0,1.25$ and $2.5 \mu \mathrm{M})$ for $24 \mathrm{~h}$ and $48 \mathrm{~h}$. Finally, the images of wound gap were taken using microscope (Olympus, Tokyo, Japan).

\section{Transwell assay}

SK-MES-1 and NCI-H520 cells were starved overnight and then cultured in the upper chamber (24-well transwell chambers, $8 \mu \mathrm{m}$ pore size, Corning, NY, USA) with serum-free medium, while high-serum medium (10\% FBS) containing dioscin was added to the lower chamber. After $48 \mathrm{~h}$ of incubation at $37^{\circ} \mathrm{C}$, cells were fixed with methanol and stained with $0.1 \%$ crystal violet. Finally, images were taken using microscope (Olympus, Tokyo, Japan). 


\section{Cell apoptosis assay}

Lung SCC cells and HBE cells were harvested and a FITC Annexin V Apoptosis Detection Kit (BD Pharmingen, NJ, USA) was used to detect cell apoptosis according to the manufacturer's protocol. Briefly, cells were washed and resuspended in binding buffer. Then, cells were stained with $5 \mu \mathrm{l}$ of FITC Annexin V and $5 \mu$ of propidium iodide (PI) followed by incubation for 15 mins at room temperature in the dark. Finally, cell apoptosis was analyzed by BD FACSVerse (BD Biosciences, San Jose, CA, USA).

\section{Analysis of mitochondrial $\Delta \Psi_{\mathrm{m}}$}

The mitochondrial $\Delta \Psi \mathrm{m}$ was detected by JC-1 Mitochondrial Membrane Potential Assay Kit (Yeasen Biotech, Shanghai, China) according to the manufacturer's instructions. After different treatments, JC-1 working solution was added to the cells and incubated at $37^{\circ} \mathrm{C}$ for 20 mins. Next, cells were washed twice with JC-1 staining buffer and analysed by BD FACSVerse (BD Biosciences, San Jose, CA, USA). And when $\Delta \Psi \mathrm{m}$ is reduced, red fluorescence decreases and green fluorescence increases. Thus, $\Delta \Psi \mathrm{m}$ variations can be reflected by the ratio of green/red fluorescence intensity.

\section{Analysis of intracellular ROS}

The intracellular ROS of cells were detected by Reactive Oxygen Species Assay Kit (Beyotime, Shanghai, China) with 2',7'-dichlorodihydrofluorescein diacetate (DCFH-DA) according to the method published previously with fine-turning[14]. Briefly, cells were harvested, washed with PBS and incubated with DCFH-DA $(0.5 \mu \mathrm{M})$ in the dark for 3 mins at $37^{\circ} \mathrm{C}$. After washing with PBS, the samples were analysed for the fluorescence of $\mathrm{DCF}$ by $\mathrm{BD}$ FACSVerse (BD Biosciences, San Jose, CA, USA).

\section{Western blotting analysis}

Cells and tissues were lysed in RIPA lysis buffer (Beyotime, Shanghai, China). Then, the protein was separated by SDS-PAGE, transferred to PVDF membranes (Millipore, Bedford, MA, USA) and probed with primary antibodies overnight at $4{ }^{\circ} \mathrm{C}$. After incubated with the corresponding secondary antibodies for $1 \mathrm{~h}$, the densities of bands were detected by ECL Chemiluminescence Kit HRP (FDbio, Hangzhou, China). Antibodies against Bcl2, Bax, cleaved caspase-3, cleaved PARP, p38, p-p38 (Thr180/ Tyr182), E-cadherin, N-cadherin and Vimentin were purchased from Cell Signalling Technology (Danvers, MA, USA). Antibodies against HSP27 and p-HSP27 (Ser15) were purchased from Diag Biotechnology (Hangzhou, China). The antibody against GAPDH was obtained from Beyotime (Shanghai, China).

\section{Xenograft model experiment}

Female BALB/c-nude mice (3-4 weeks) were purchased from Shanghai Experimental Animal Center (Chinese Academy of Sciences, Shanghai, China). $8 \times 10^{5}$ suspended NCI-H520 cells (in $100 \mu \mathrm{l}$ PBS) were injected into the right back of each mouse. When tumour volumes reached approximately 50-100 $\mathrm{mm}^{3}$, the mice were randomized into 2 groups (6 mice per group). Each group of mice was given with $0.5 \%$ Sodium carboxymethylcellulose (Sangon Biotech, Shanghai, China) or dioscin $(80 \mathrm{mg} / \mathrm{kg} /$ day $)$ by gavage for 12 days. Tumours were measured every 3 days using a vernier calliper, and the tumour volume was calculated by the following formula: $\mathrm{V}=$ (Length ${ }^{*}$ Width $\left.{ }^{2}\right) / 2$. At the endpoint, mice were sacrificed and the tumours were collected for further analysis. The experiments were performed according to the Regulations for the Administration of Affairs Concerning Experimental Animals and were approved by the Experimental Animal Ethics Committee of Zhejiang University.

\section{TUNEL assay}

Apoptosis in tissues was detected by One Step TUNEL Apoptosis Assay Kit (Beyotime, Shanghai, China), according to the instruction provided with the kit. Briefly, the tissue sections were dewaxed, rehydrated, and permeabilized with $20 \mu \mathrm{g} / \mathrm{ml}$ proteinase $\mathrm{K}$ solution for $20 \mathrm{mins}$ at $37{ }^{\circ} \mathrm{C}$. After washed with PBS, the tissue sections were incubated with TUNEL reaction mixture in the dark for $1 \mathrm{~h}$ at 37 ${ }^{\circ} \mathrm{C}$. The nuclei were stained with 4',6-diamidino-2phenylindole (DAPI). After washed with PBS, the tissue sections were mounted with antifade mounting medium (Beyotime, Shanghai, China) and images were taken using microscope (Olympus, Tokyo, Japan).

\section{Statistical analysis}

All data were presented as the mean \pm SD of three independent experiments. The statistical significance was assessed by Student's t-test for two groups or one-way ANOVA for multiple groups using Prism 6.04 software (GraphPad Software Inc., San Diego, CA, USA). ${ }^{*} p<0.05,{ }^{* *} p<0.01,{ }^{* * *} p<0.001$ are determined as significance.

\section{Results}

\section{Dioscin inhibited cell proliferation in lung SCC cells}

Lung SCC cells and HBE cells were treated with dioscin $(0,1.25,2.5,5,10 \mu \mathrm{M})$ for 24 and $48 \mathrm{~h}$. As shown in Fig. 1A, the viability of lung SCC cells was 
significantly inhibited by dioscin in a dose-dependent manner, with IC50 values of $4.59 \mu \mathrm{M}$ (NCI-H520) and $2.05 \mu \mathrm{M}$ (SK-MES-1) at $48 \mathrm{~h}$. The IC50 value of HBE cells after $48 \mathrm{~h}$ of treatment was $8.47 \mu \mathrm{M}$, indicating that the effect of dioscin was more potent in lung SCC

A

Cell viability of SK-MES-1

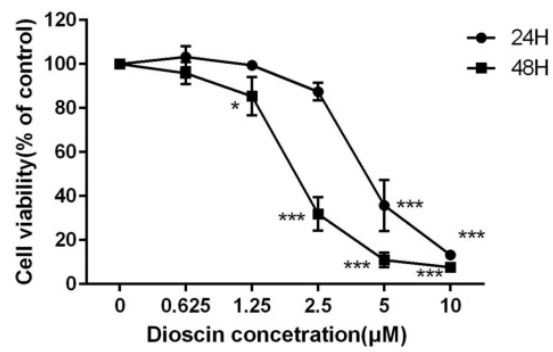

Cell viability of HBE

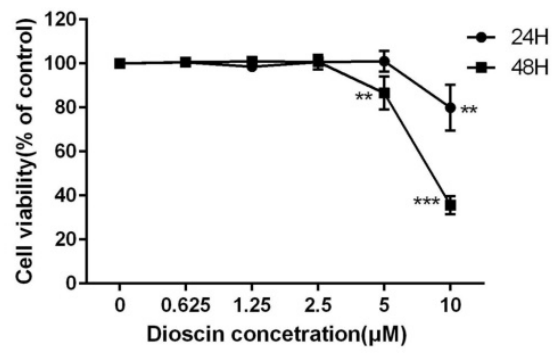

B

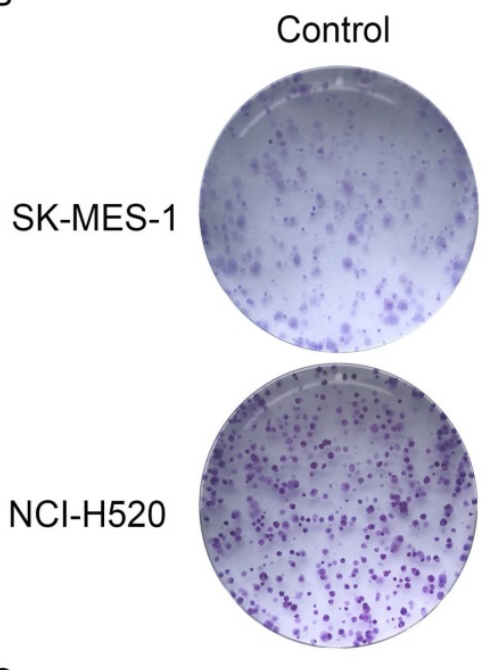

$\operatorname{Dioscin}(1.25 \mu \mathrm{M})$
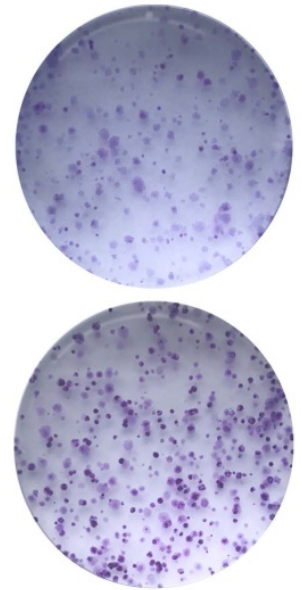

C

SK-MES-1

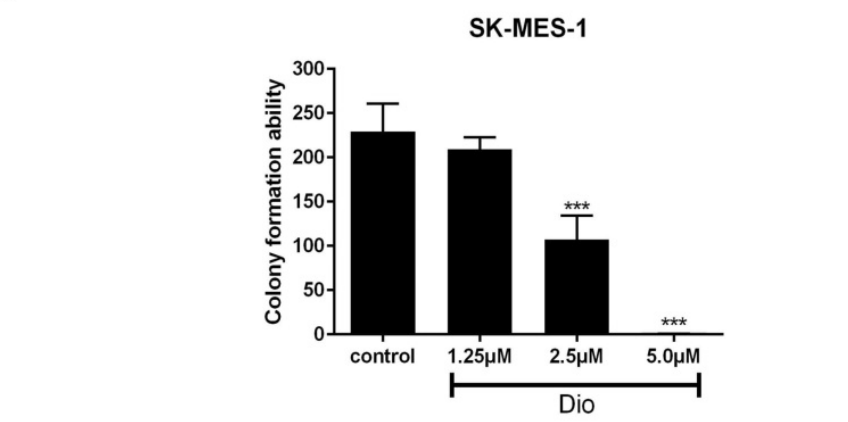

cells than in HBE cells (Fig. 1A). A colony formation assay was performed to determine the effect of dioscin on lung SCC cell proliferation. We found that dioscin substantially decreased the colony numbers in both NCI-H520 and SK-MES-1 cells (Fig. 1B and 1C).

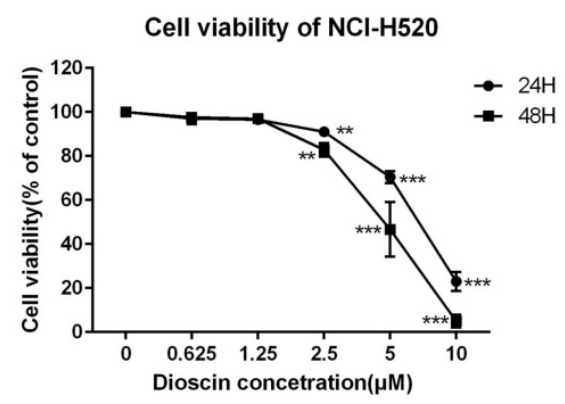

\begin{tabular}{cc}
\multicolumn{2}{c}{ IC50 of dioscin in cells $(48 \mathrm{H})$} \\
\hline Cell lines & IC50 $(\mu \mathrm{M})$ \\
\hline SK-MES-1 & 2.04 \\
NCI-H520 & 4.59 \\
HBE & 8.47 \\
\hline
\end{tabular}
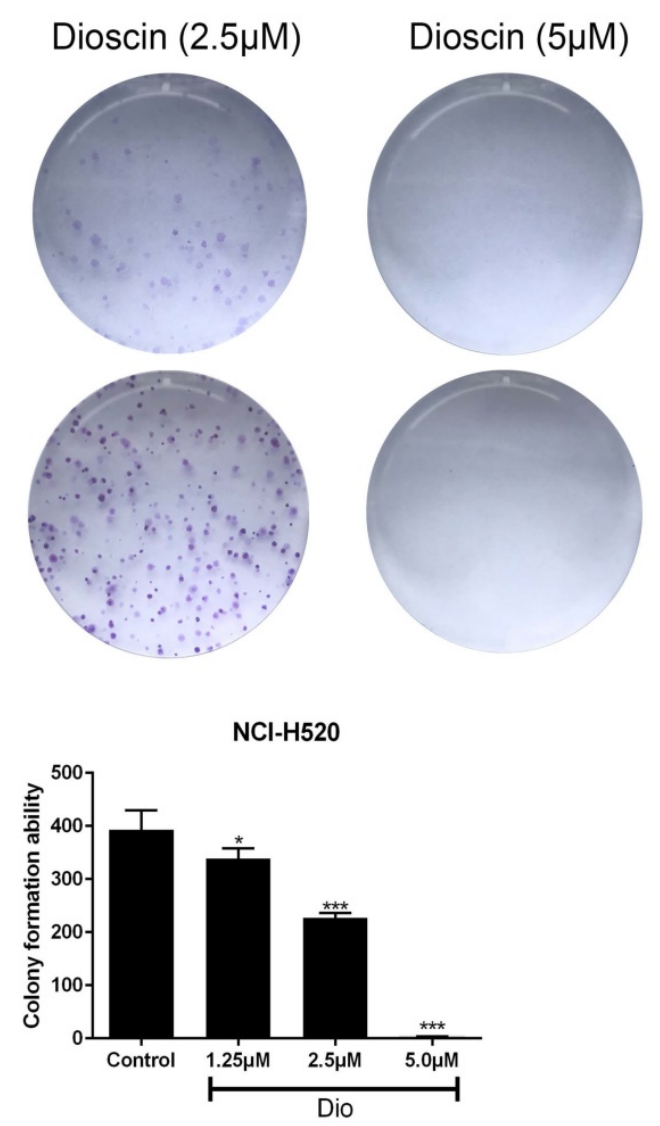

Figure 1. Dioscin inhibited cell proliferation in lung SCC cells. (A) SK-MES-1, NCI-H520 and HBE cells were treated with Dio $(0,1.25,2.5,5,10 \mu M)$ for $24 \mathrm{~h}$ and $48 \mathrm{~h}$. Cell viability was determined by CCK-8 assay, which was performed in triplicate with three independent experiments. The IC50 of dioscin at $48 \mathrm{~h}$ in each cell line was calculated. (B, C) Dioscin suppressed SK-MES- 1 and NCl-H520 cells colony formation. Cells were treated with Dio $(0,1.25,2.5,5 \mu \mathrm{M})$. After 7-10 days, the cells were stained with $0.1 \%$ crystal violet. The data are representative of three independent experiments and are presented as the mean \pm SD. Significant differences compared with the control are indicated by $* p<0.05, * * p<0.01$, and ${ }^{* * *} p<0.001$ 
A

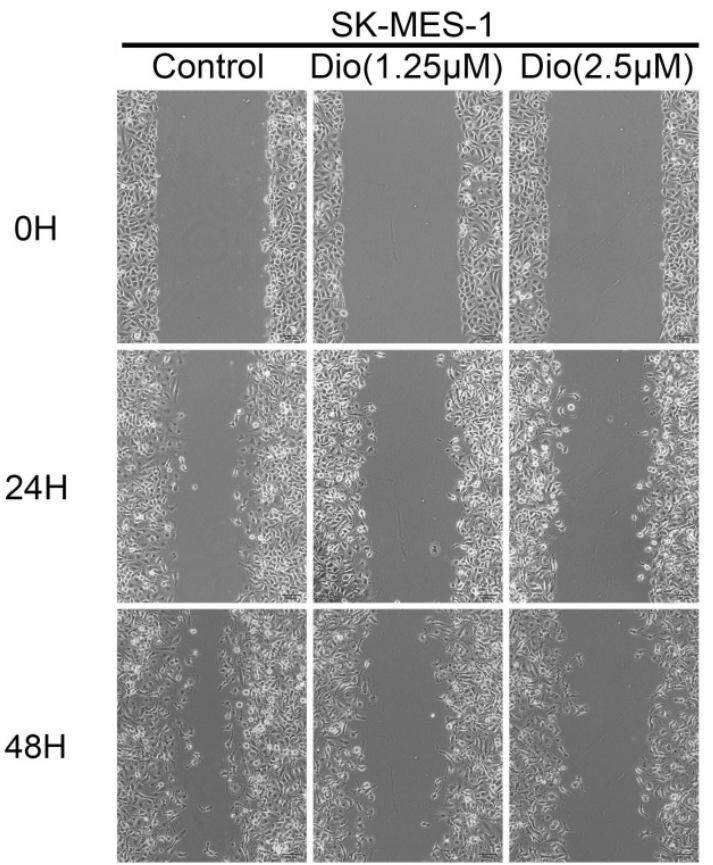

B
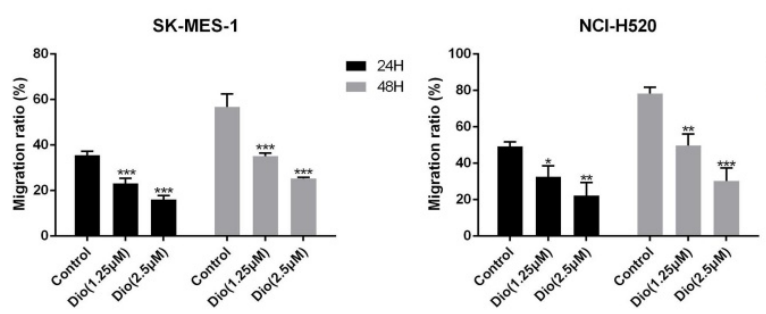

D
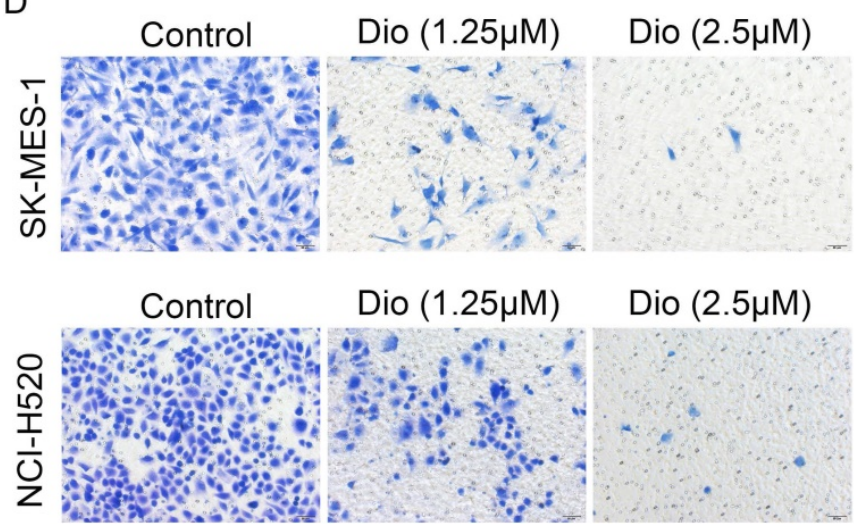

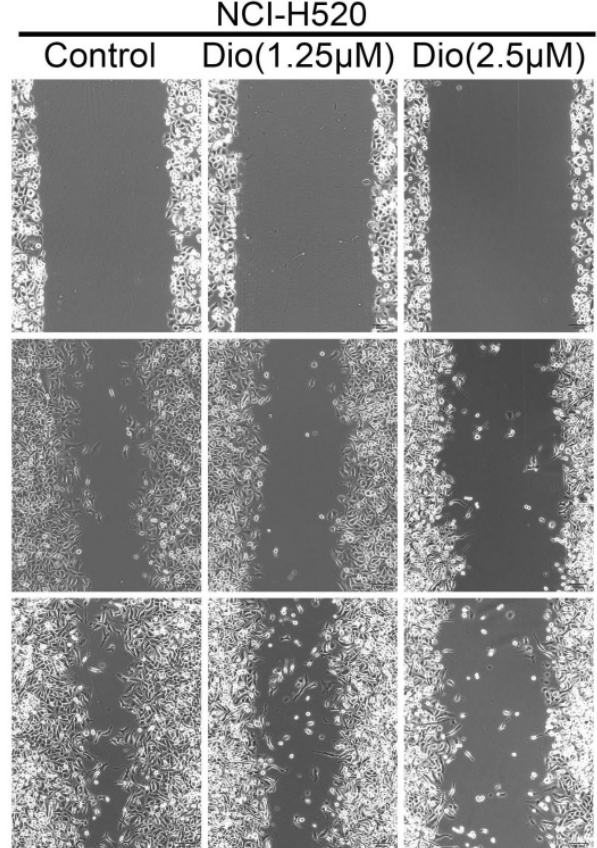

C

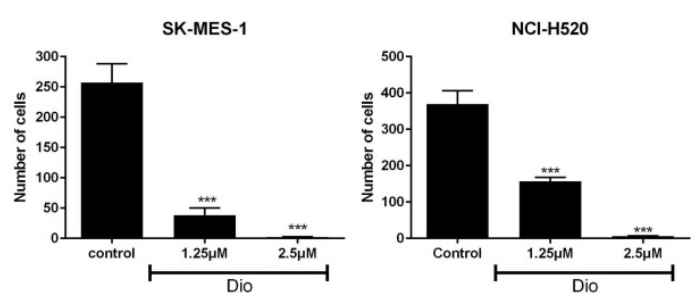

E

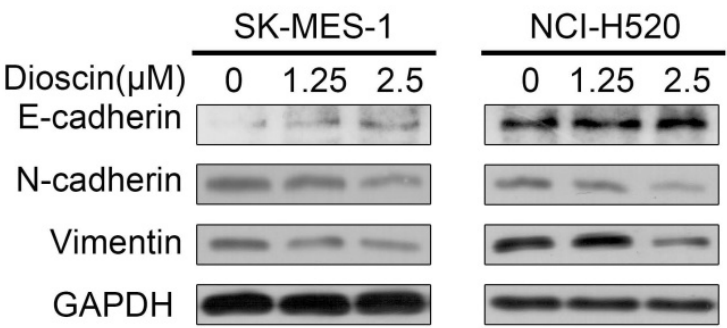

Figure 2. Dioscin suppressed the cell migration of lung SCC cells. SK-MES-1 and NCl-H520 cells were treated with Dio $(0,1.25,2.5 \mu M)$ for $48 \mathrm{~h}$. The effect of dioscin on cell migration was assessed by wound healing assay (scale bar=100 $\mu \mathrm{m})(\mathrm{A}, \mathrm{B})$ and Transwell assay (scale bar=50 $\mu \mathrm{m})(\mathrm{C}, \mathrm{D})$. The data are representatives of three independent experiments and presented as the mean \pm SD. Significant differences compared with the control are indicated by $* p<0.05$, $* * p<0.01$, and $* * * p<0.001$. $(E)$ The expression levels of E-cadherin, $\mathrm{N}$-cadherin and Vimentin were analysed by western blot.

\section{Dioscin suppressed cell migration in lung SCC cells}

Wound healing and Transwell assays were used to determine the impact of dioscin on cell migration. As shown in Fig. 2A and 2B, dioscin treatment (1.25 and $2.5 \mu \mathrm{M}$ ) resulted in slower rates of wound healing. In addition, dioscin markedly reduced the numbers of NCI-H520 and SK-MES-1 cells moving to the lower chamber (Fig. 2C and 2D). Epithelial-tomesenchymal transition (EMT) is a program which enhances the migration of tumour cells [26]. So, we also detected the expression changes of EMT-related proteins. And we found that dioscin mildly downregulated the expression levels of $\mathrm{N}$-cadherin and Vimentin, and up-regulated the expression levels 
E-cadherin (Fig. 2E). Therefore, dioscin could suppress the migration of lung SCC cells.

\section{Dioscin induced cell apoptosis in lung SCC cells}

NCI-H520, SK-MES-1 and HBE cells were treated with dioscin for $48 \mathrm{~h}$ and cell apoptosis was detected by PI/FITC- Annexin V double staining. Our data revealed that dioscin had a significant dosedependent effect on apoptotic inducement in lung
SCC cells, while the effect in HBE cells was not obvious (Fig. $3 \mathrm{~A}$ and $3 \mathrm{~B}$ ). A reduction in $\Delta \Psi \mathrm{m}$ often marked the early stage of mitochondrial apoptosis. Compared with the control group, dioscin treatment increased the ratio of green/red fluorescence intensity in both NCI-H520 and SK-MES-1 cells (Fig. 3C). Then, we detected the expression of $\mathrm{B}$ cell lymphoma 2 (Bcl2) and its family members to explore how dioscin influences cell apoptosis. We found that dioscin downregulated the expression of $\mathrm{Bcl} 2$ and

A

B
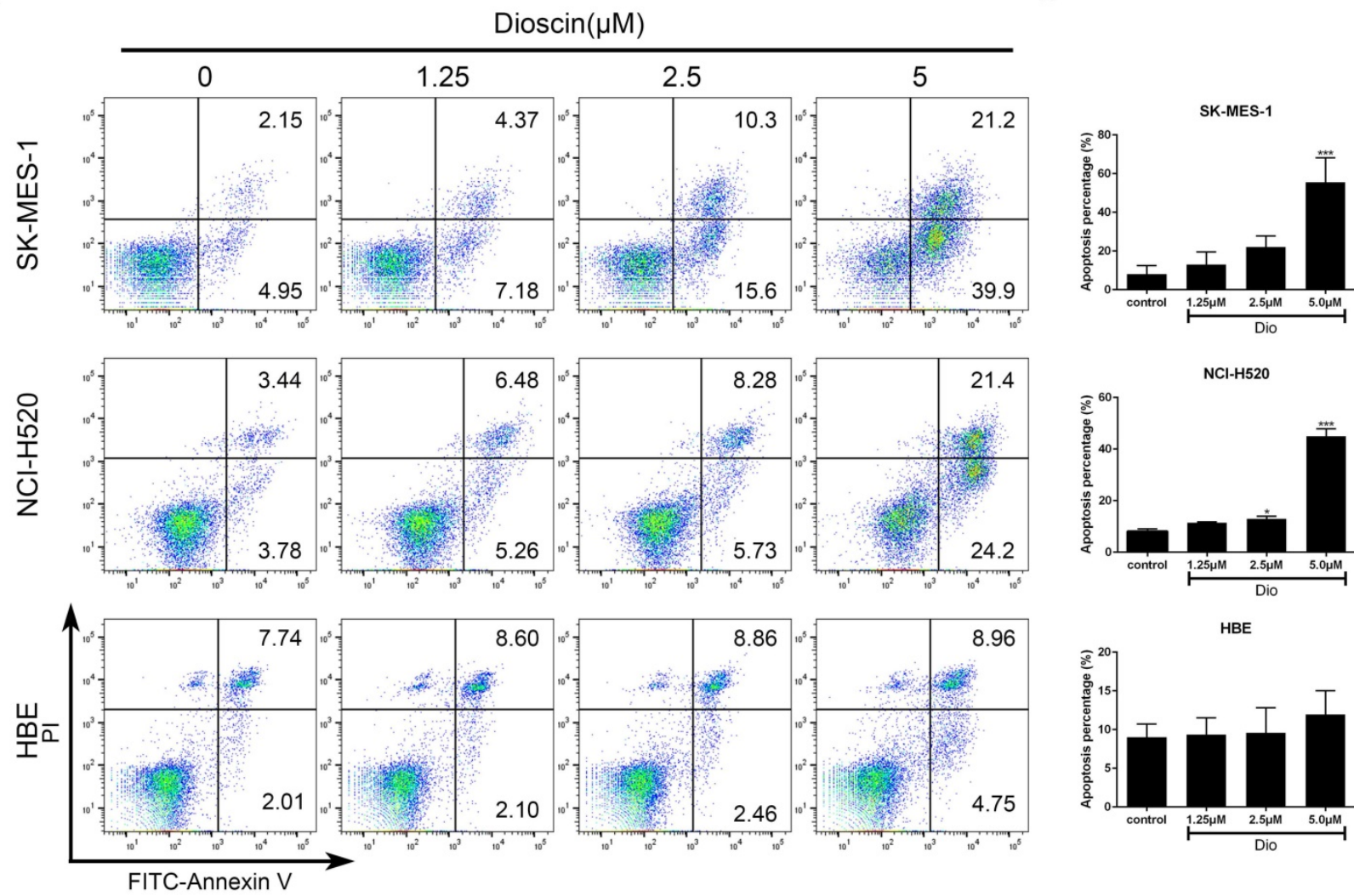

C

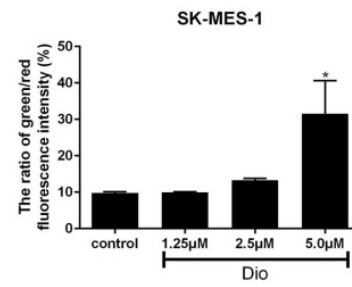

D
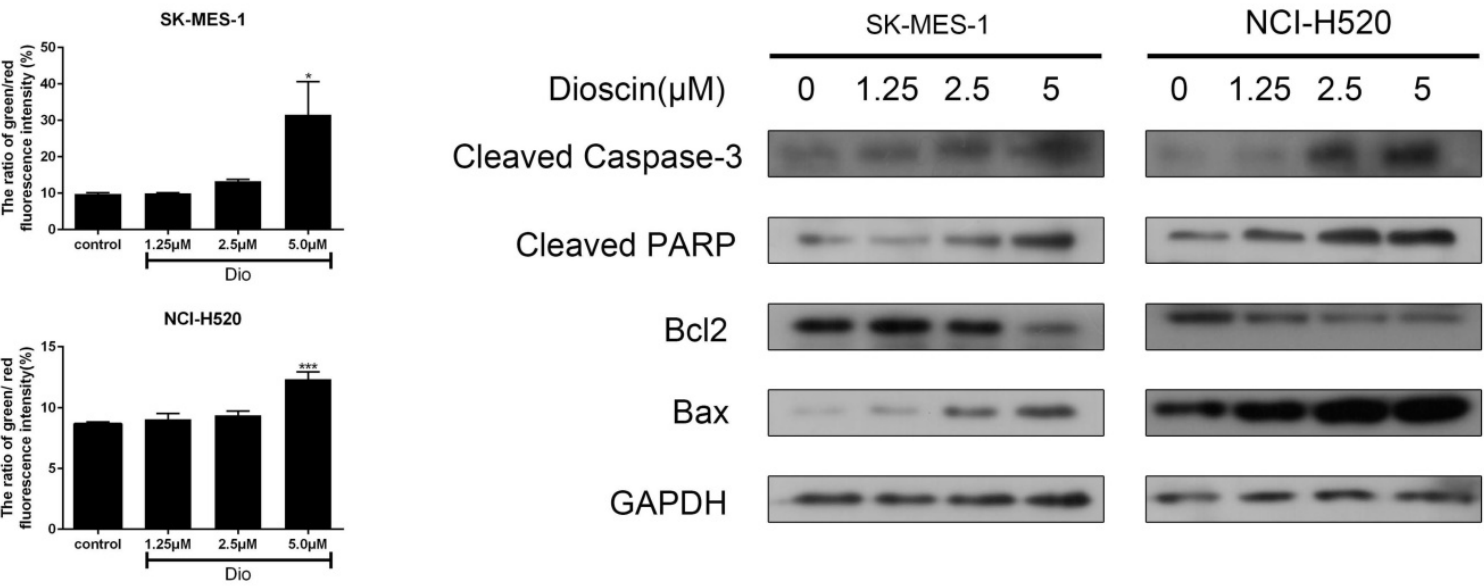

Figure 3. Dioscin induced cell apoptosis in lung SCC cells. NCI-H520, SK-MES-1 and HBE cells were treated with dioscin (0, 1.25, 2.5, $5 \mu$ M) for 48 h. (A, B) Cell apoptosis was measured by flow cytometry. (C) The changes in $\Delta \Psi_{\mathrm{m}}$ were monitored by JC-1 staining and the ratio of green/red fluorescence intensity was used to calculate mitochondrial depolarization. Data are the mean \pm SD of triplicate samples. Significant differences compared with the control are indicated by $* p<0.05$, $* * p<0.01$, and $* * * p<0.001$. (D) The expression levels of cleaved caspase-3, cleaved PARP, Bax and Bcl-2 were analysed by western blot. 
upregulated the expression of Bax (Fig. 3D). Moreover, caspase- 3 and its downstream target PARP, which indicate the point at which the extrinsic and intrinsic apoptotic pathways merge, were obviously activated with dioscin treatment (Fig. 3D).

\section{The p38-MAPK/HSP27 signalling pathway was involved in apoptosis induced by dioscin}

We further evaluated the effect of dioscin on the p38-MAPK/HSP27 pathway in lung SCC cells. As shown in Fig. 4A, we found that dioscin clearly increased the levels of p-p38 and p-HSP27, while the levels of total p38 and HSP27 were not obviously changes in NCI-H520 and SK-MES-1 cells. In addition, to ascertain whether the p38-MAPK/HSP27 signalling pathway was involved in dioscin-induced apoptosis, we pre-treated the cells with p38-MAPK activator Anisomycin and p38-MAPK inhibitor SB203580. Anisomycin pre-treatment activated p38 and HSP27 and induced apoptosis (Fig. 4B and 4C),
A

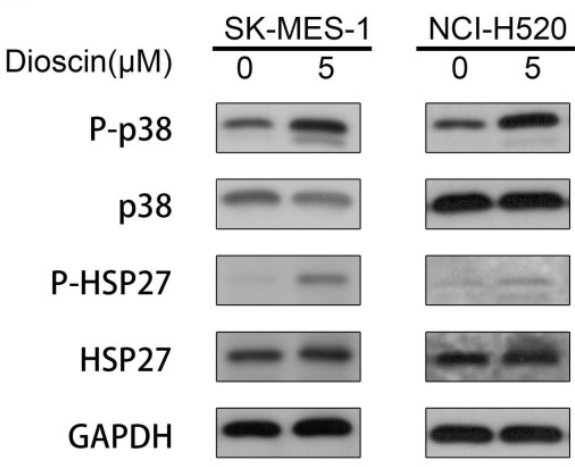

B

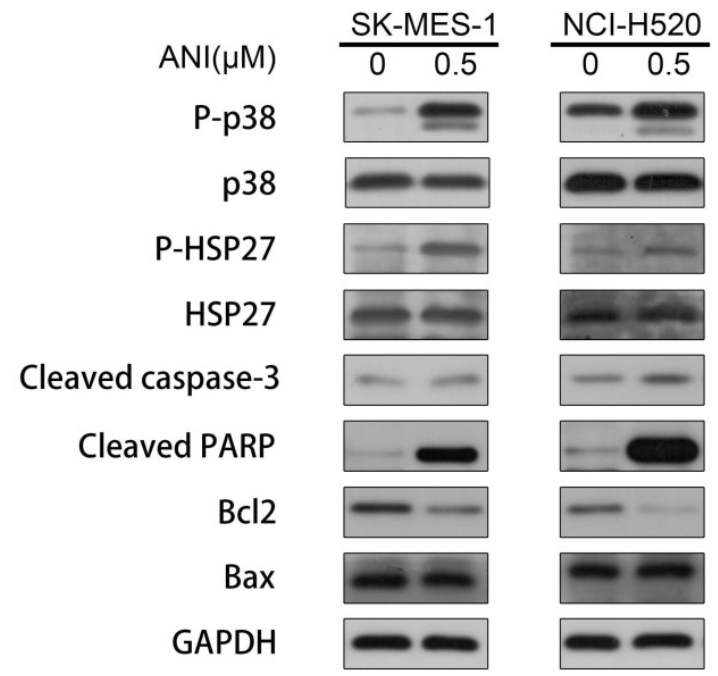

C

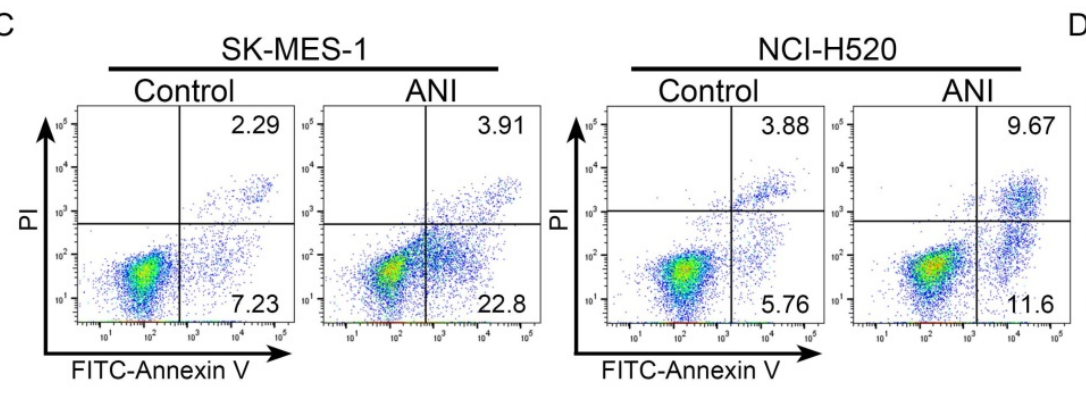

D

E
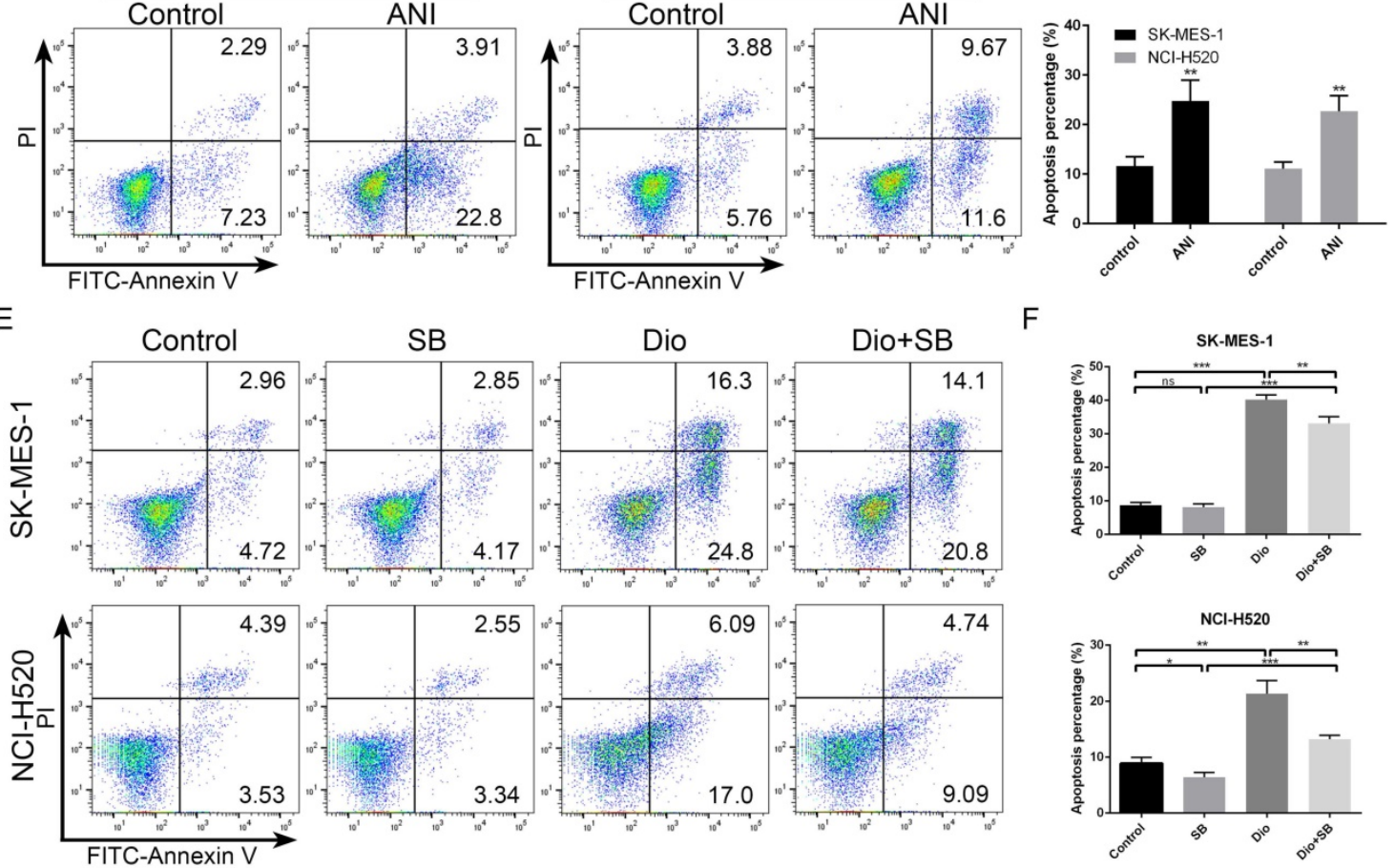

Figure 4. The p38-MAPK/HSP27 signalling pathway was involved in dioscin-induced apoptosis. (A) NCl-H520 and SK-MES- 1 cells were treated with dioscin (5 $\mu$ M) for $48 \mathrm{~h}$. The levels of p-p38, p38, p-HSP27 and HSP27 were analysed by western blotting. (B, C) NCl-H520 and SK-MES- 1 cells were treated with Anisomycin (0.5 $\mu$ M) for 24 h. Then, cell apoptosis was measured by flow cytometry, and the expression levels of p-p38, p38, p-HSP27, HSP27, Bax, Bcl2, cleaved caspase-3 and cleaved PARP were analysed by western blotting. (D) NCl-H520 and SK-MES-1 cells were pretreated with SB203580 (5 $\mu \mathrm{M})$ for $2 \mathrm{~h}$ before exposure to dioscin for $48 \mathrm{~h}$. Then, cell apoptosis was measured by flow cytometry. Data are presented as the mean \pm SD of triplicate samples. $* P<0.05$, $* * P<0.01$, and $* * * P<0.001$ vs control. 
while SB203580 partly reversed dioscin-induced apoptosis (Fig. 4D). In conclusion, the p38MAPK/HSP27 signalling pathway promoted dioscinmediated apoptosis.

\section{Intracellular ROS accumulation was a pivotal event in dioscin-induced apoptosis}

To demonstrate whether ROS was involved in dioscin-induced lung SCC cell apoptosis, we analysed the intracellular ROS generation in lung SCC cells after dioscin treatment. As shown in Fig. 5A and 5B, dioscin elevated intracellular ROS levels in NCI-H520 and SK-MES-1 cells. Then, NAC, an ROS inhibitor antioxidant, was used to pre-treat lung SCC cells. We found NAC markedly attenuated dioscin-induced ROS accumulation and cell apoptosis in lung SCC
A

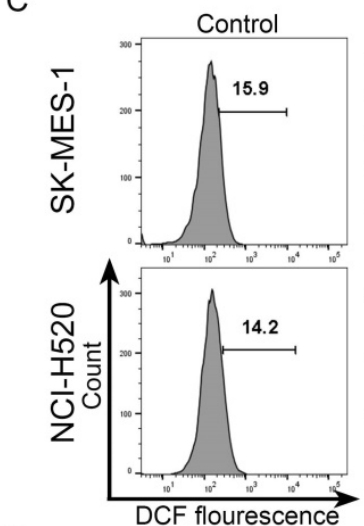

E

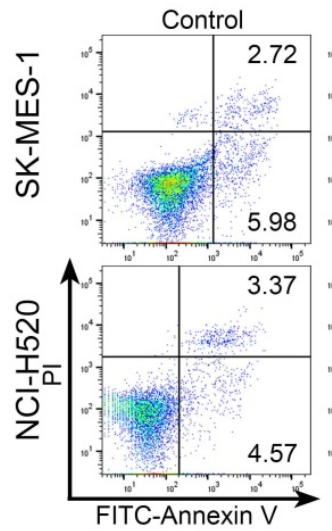

G
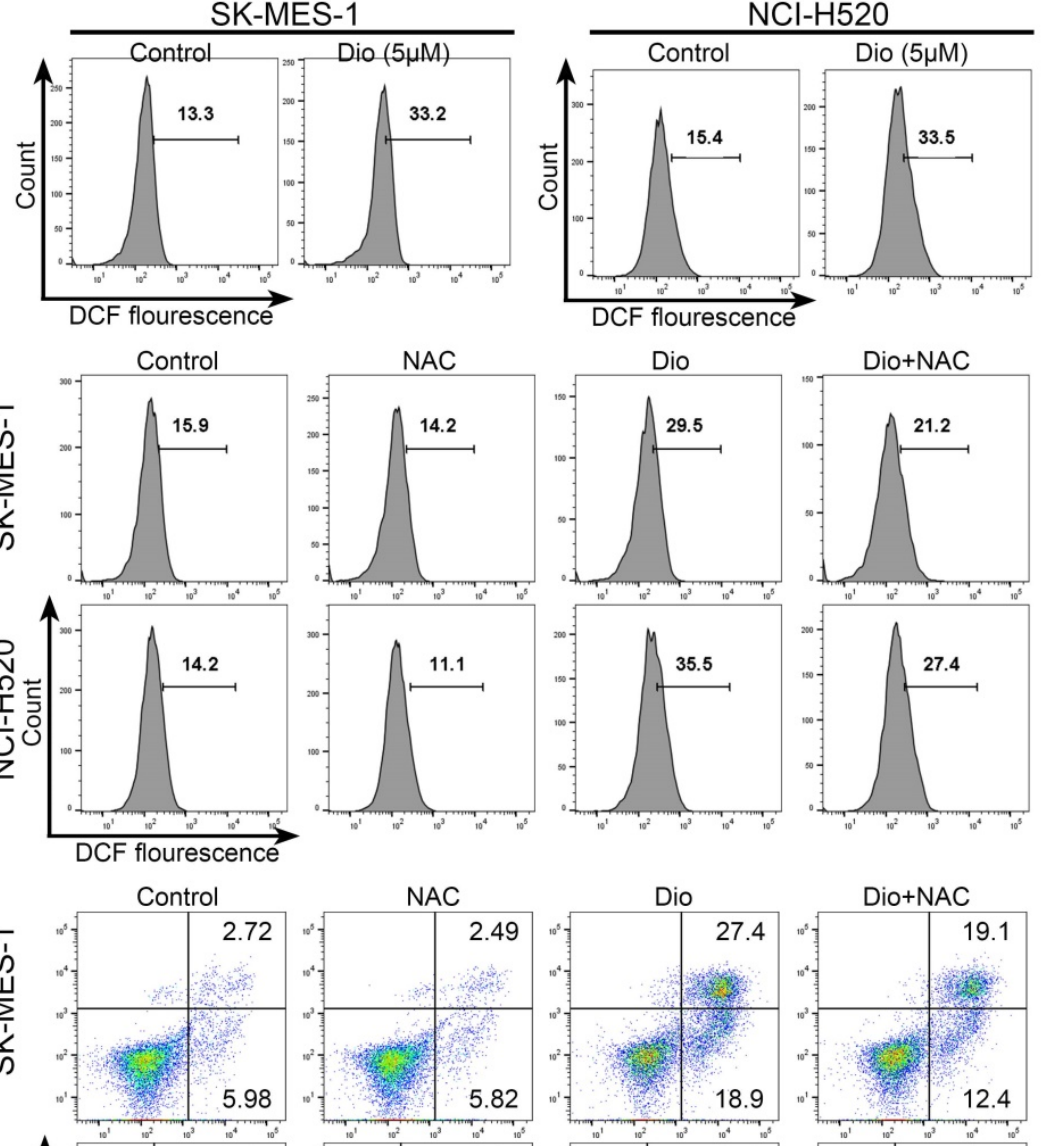

F

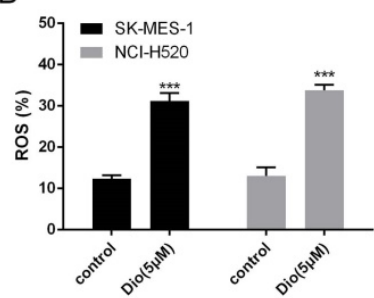

D
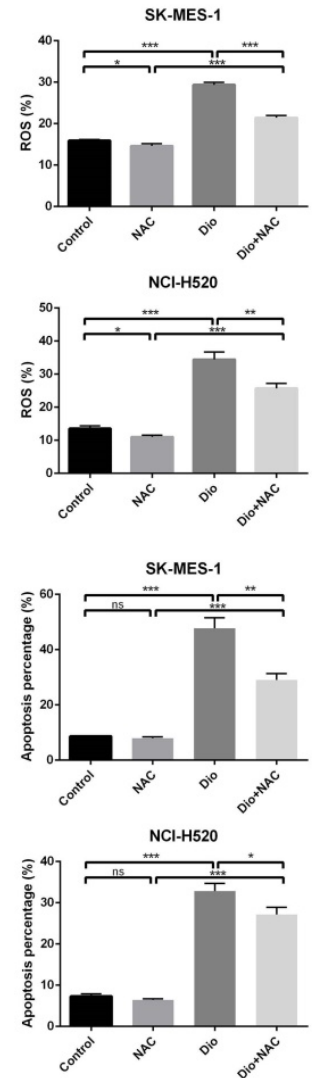

$\mathrm{H}$
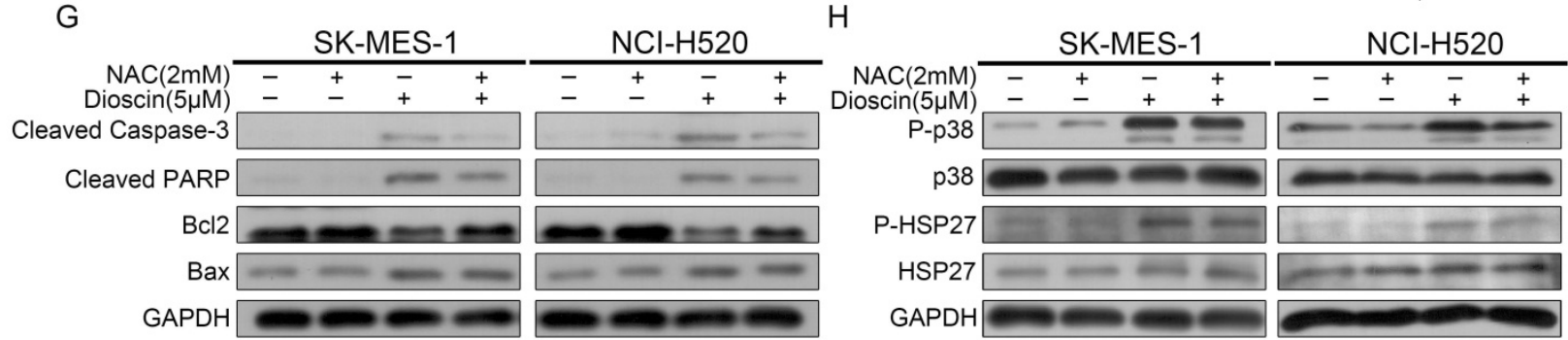

Figure 5. Intracellular ROS accumulation was a pivotal event in dioscin-induced apoptosis. (A, B) NCl-H520 and SK-MES- 1 cells were treated with dioscin (5 $\mu$ M) for 48 h. The intracellular ROS levels were measured by flow cytometry. Data are presented as the mean $\pm \mathrm{SD}$ of triplicate samples. $* p<0.05$, $* * p<0.01$, and $* * * p<0.001$ vs control. $(\mathrm{C}-\mathrm{H})$ $\mathrm{NCl}-\mathrm{H} 520$ and SK-MES- 1 cells were pretreated with NAC $(2 \mathrm{mM})$ for $24 \mathrm{~h}$ before exposure to dioscin for $48 \mathrm{~h}$. Then, cell apoptosis and intracellular ROS levels were measured by flow cytometry. Data are presented as the mean \pm SD of triplicate samples. ${ }^{*} p<0.05,{ }^{*} p<<0.01$, and ${ }^{* * *} p<0.001$ vs control. The expression levels of cleaved caspase 3 , cleaved PARP, Bax, Bcl2, P-p38, p38, p-HSP27 and HSP27 were analysed by western blotting. 
cells (Fig. 5C-G). Moreover, NAC combined with dioscin decreased the levels of p-p38 and p-HSP27 compared to those with dioscin treatment alone (Fig. $5 \mathrm{H})$. Therefore, we considered that dioscin may trigger the ROS-mediated p38-MAPK/HSP27 signalling pathway, leading to cell apoptosis.

\section{Dioscin exerted an anti-tumour effect in NCl-H520 xenograft models}

In the present study, we constructed NCI-H520 xenograft models to confirm the anti-tumour effect of dioscin in vivo. As shown in Fig. 6A, there was a significant reduction in tumour volume in dioscintreated mice compared with vehicle-treated control mice at day 12. At the endpoint of the experiment, the mice were sacrificed. Tumours were harvested, and the tumour weights were measured. The weight of NCI-H520 tumours in dioscin-treated mice was lower than that in control mice (Fig. 6B and 6C). Then, we detected apoptosis in the xenograft tumours. The
A

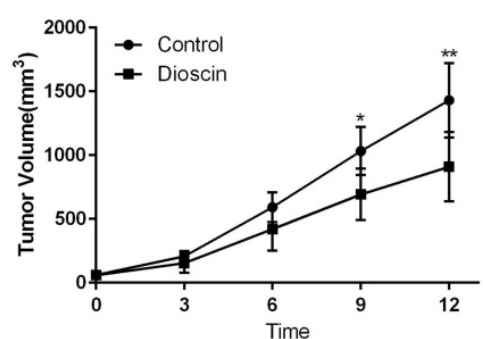

B

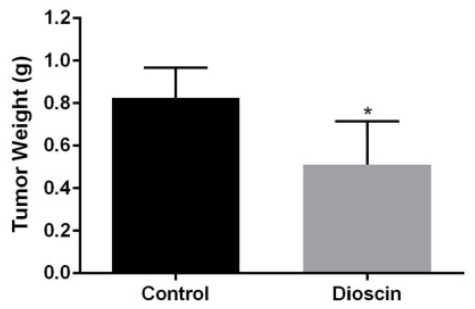

C

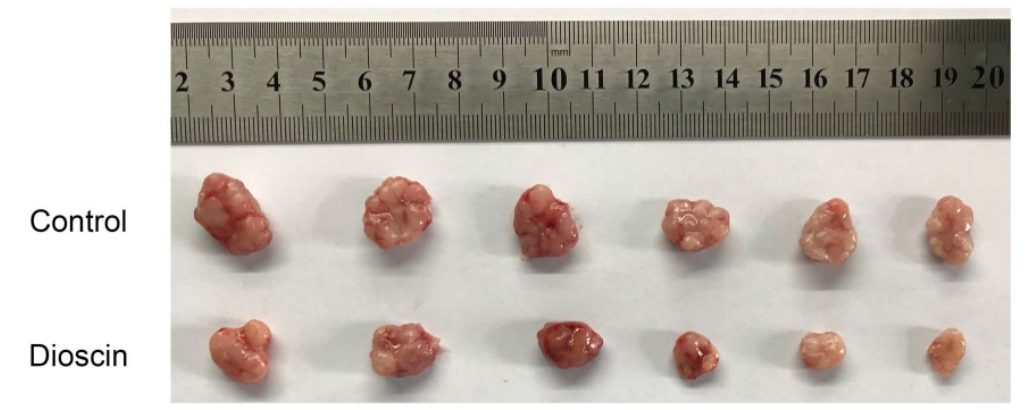

D

DAPI

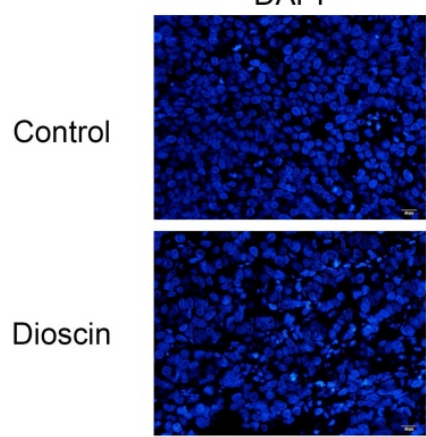

E

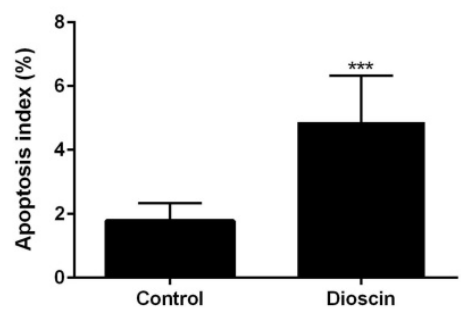

TUNEL
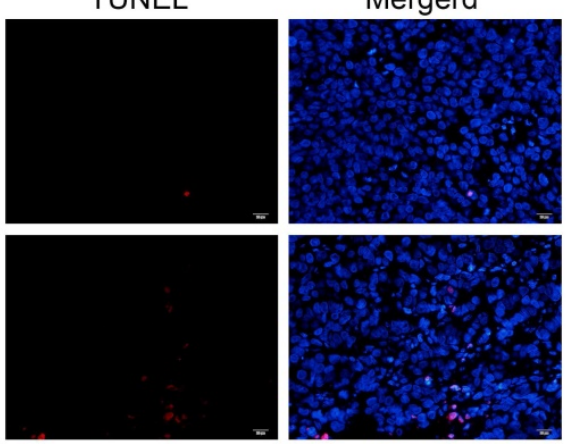

F

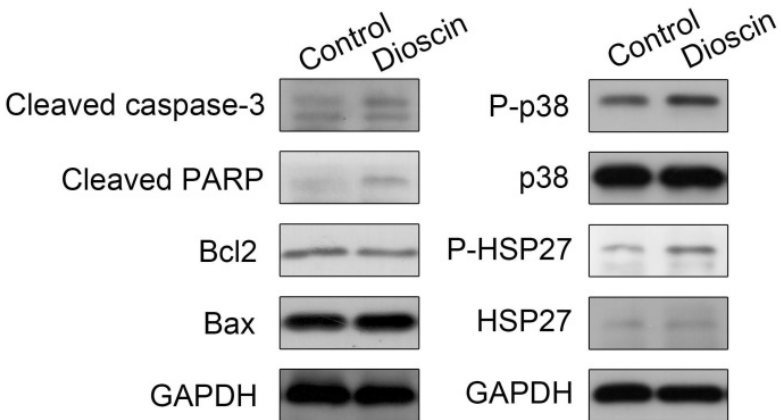

Figure 6. In vivo anti-tumour effects of dioscin in xenograft models. $\mathrm{NCl}-\mathrm{H} 520$ cells were subcutaneously injected into the backs of BALB/c nude mice. When the tumour reached a volume of 50-100 $\mathrm{mm}^{3}$, mice were divided into two groups and orally treated with vehicle or dioscin $(80 \mathrm{mg} / \mathrm{kg} / \mathrm{d})$ for $12 \mathrm{days}(\mathrm{n}=6)$. (A) The tumour volumes of the mice were determined every three days after the onset of treatment. (B, C) On day 12, the tumours were carefully dissected from the mice and the weights of tumours was measured. (D, E) Apoptotic cells in tumour samples were detected by TUNEL assay (scale bar $=20 \mu \mathrm{m})$. Data are presented as the mean \pm SD. Significant differences compared with the control are indicated by $* p<0.05$, $* * 00.01$, and $* * * p<0.001$. (F) The harvested tumours were subsequently lysed and western blot analysis was performed for cleaved caspase-3, cleaved PARP, Bax, Bcl2, p-p38, p38, p-HSP27, HSP27 and GAPDH expression. 
TUNEL assay showed that the number of apoptotic cells was higher in the dioscin treatment group than in the control group (Fig. 6D and 6E). Moreover, the expression levels of cleaved caspase- 3 and cleaved PARP in the dioscin treatment group were also increased (Fig. 6F). To evaluate whether the inhibition of lung SCC tumour xenograft growth by dioscin was associated with the p38-MAPK/HSP27 signalling pathway, we determined the levels of p38, p-p38, HSP27, p-HSP27 in tumour samples by western blotting. Here, we found that dioscin treatment activated the p38-MAPK/HSP27 signalling pathway in tumour tissue and these results were similar to the results in vitro (Fig. 6F).

\section{Discussion}

Though multimodality therapies have been developed, lung SCC is still a highly aggressive disease with limited benefit from current treatments. Therefore, the identification of novel adjuvant treatments for lung SCC is still urgent. Recent studies have shown the powerful anti-tumour effect of dioscin in different cancer [6]. In lung cancer, it has been proven that dioscin inhibits TGF $\beta 1$-induced cell migration and invasion [27]. A previous study also found that dioscin suppresses lung cancer growth by inducing DNA damage and activating mitochondrial apoptosis [11]. Moreover, targeted liposomes loaded with both daunorubicin and dioscin inhibit metastasis of lung cancer [28]. More importantly, dioscin overcomes tyrosine kinase inhibitor resistance by down-regulating phosphatase $\mathrm{SH} 2$ domaincontaining phosphatase-2 (SHP2) expression in EGFRmutated lung adenocarcinoma cells [29]. However, few studies have focused on the antineoplastic activity of dioscin in lung SCC. The present study indicated that dioscin inhibited lung SCC growth both in vitro and in vivo. The anti-tumour effects of dioscin were mainly associated with signalling pathways involving the accumulation of ROS and the activation of p38-MAPK and its downstream target HSP27.

Uncontrolled cell growth and migration are two of the biological behaviours of malignant cells that cause poor prognosis in patients with lung cancer [12, 27]. Our results showed that dioscin obviously inhibited cell proliferation and cell migration in lung SCC cells, which was similar to the results of previous studies [6, 8, 12]. NCI-H520 xenograft model experiments also showed the significant anti-tumour activity of dioscin. The tumour volume and tumour weight of mice receiving dioscin treatment were apparently decreased compared to those of control mice. In accordance with previous studies, we found that dioscin induced cell apoptosis in lung SCC cells, regardless of whether the apoptosis was in the early stage or late stage, with a marked upregulation expression of cleavage of caspase- 3 and PARP [6, 8, 17]. Moreover, the results of xenograft models verified the pro-apoptotic effect of dioscin in vivo. Importantly, our study further indicated that dioscininduced cell apoptosis was related to ROS.

It has been reported that the regulation of intracellular redox status by ROS is related to apoptosis by influencing intracellular signalling pathways [30, 31]. Fluctuations in intracellular ROS levels induced by dioscin cause damage to cellular structures, which accelerating apoptosis [15]. ROS generation is explicated to induce apoptosis in human lung SCC cells [31]. In the present study, we noted that the accumulation of intracellular ROS was triggered by dioscin in lung SCC cells. In addition, the ROS scavenger NAC exerted strong effects not only by decreasing dioscin-induced intracellular ROS but also by attenuating dioscin-induced apoptosis. Hence, we elucidated dioscin-induced apoptosis in lung SCC cells by upregulating intracellular ROS levels.

ROS has been demonstrated to regulate p38-MAPK, which extensively participates in the

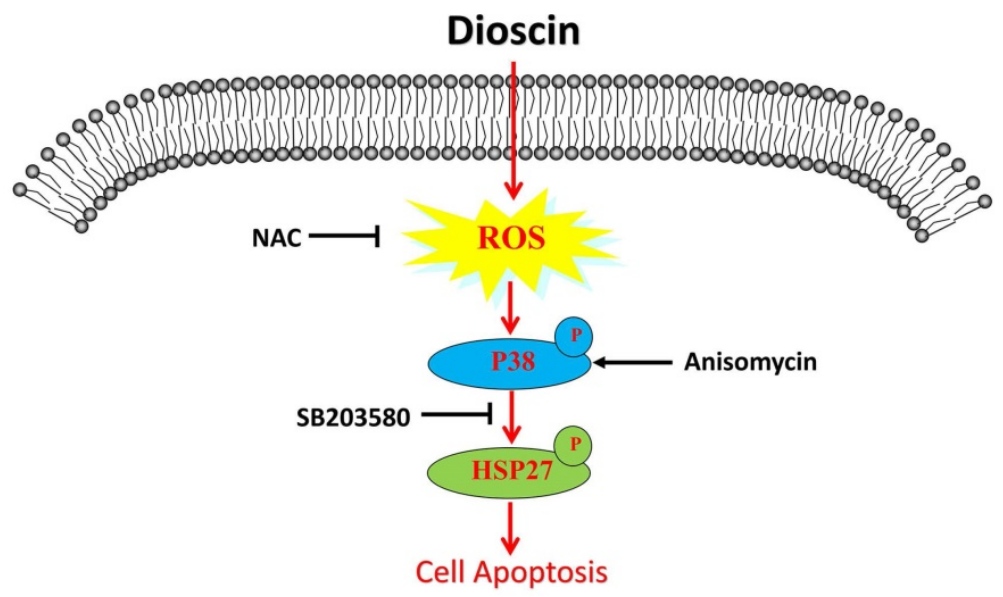

Figure 7. Schematic illustration of dioscin-induced apoptosis in lung squamous cell carcinoma. 
regulation of cell apoptosis, proliferation and differentiation [14, 32]. In colon cancer cells, dioscin activates the p38-MAPK signalling pathway to induce ROS-mediated apoptosis [17]. However, some controversial evidence has proven that inactivation of p38-MAPK inhibits tumorigenesis [33, 34]. HSP27 is one of the downstream targets of p38-MAPK and high levels of HSP27 enhance the malignant behaviour of tumour cells, including over-proliferation, metastasis and less apoptosis $[35,36]$. In human lung cancer, low expression of HSP27 is associated with better prognosis [37]. However, the role of phosphorylated HSP27 in apoptosis remains ambiguous [24]. In the present study, we observed that the expression of phosphorylated p38-MAPK in lung SCC was increased with upregulation of phosphorylated HSP27 after treatment with dioscin both in vivo and in vitro. The activation of p38-MAPK/HSP27 induced by the p38-MAPK activator Anisomycin caused the apoptosis of lung SCC cells. On the other hand, the p38-MAPK inhibitor SB203580 attenuated dioscininduced apoptosis. Thus, we concluded that dioscin induces apoptosis through the p38-MAPK/HSP27 signalling pathway. Similar to our findings, activation of p38-MAPK/HSP27 induces brain endothelial cell apoptosis [24]. A recent study found that activation of p38-MAPK/HSP27 suppresses stem cell-like properties in human non-small cell lung cancers, which indicates a new function of p38-MAPK in tumour suppression [38]. To further investigate whether ROS regulates the p38-MAPK/HSP27 pathway in lung SCC cells, we treated the cells with NAC and found that NAC restrains dioscin-induced activation of p38-MAPK/HSP27. Above all, we illustrated that intracellular ROS regulated the activation of p38-MAPK/HSP27 in the dioscininduced apoptosis of lung SCC cells in vitro.

\section{Conclusion}

In summary, our findings demonstrate that dioscin facilitates ROS-induced apoptosis via the p38-MAPK/HSP27-mediated pathways in lung SCC cells and suggested that dioscin might be used as a potential anticancer agent in lung SCC treatment.

\section{Abbreviations}

SCC: squamous cell carcinoma; ROS: reactive oxygen species; NAC: N-acetyl-L-cysteine; HSP27: heat shock protein 27; FBS: fetal bovine serum; DMSO: dimethyl sulfoxide; DCFH-DA: 2',7'-dichlorodihydrofluorescein diacetate; DAPI: 4',6-diamidino-2phenylindole; EMT: epithelial-to-mesenchymal transition; Bcl2: B cell lymphoma 2; SHP2: SH2 domain-containing phosphatase-2.

\section{Acknowledgments}

This work was supported by the key research and development program of Zhejiang Province under Grant Number 2019C03042; National Natural Science Foundation of China under Grant Number 81670017; Natural Science Foundation of Zhejiang Province under Grant Number Y18H160054 and Q18H160029; and Project of Health and Family Planning Commission of Zhejiang Province, China under Grant Number 2015KYA085.

\section{Competing Interests}

The authors have declared that no competing interest exists.

\section{References}

1. Bray F, Ferlay J, Soerjomataram I, Siegel RL, Torre LA, Jemal A. Global cancer statistics 2018: GLOBOCAN estimates of incidence and mortality worldwide for 36 cancers in 185 countries. CA Cancer J Clin. 2018; 68: 394-424.

2. Chen W, Zheng R, Baade PD, Zhang S, Zeng H, Bray F, et al. Cancer statistics in China, 2015. CA Cancer J Clin. 2016; 66: 115-32.

3. Koutsoukos K, Mountzios G. Novel therapies for advanced squamous cell carcinoma of the lung. Future oncol. 2016; 12: 659-67.

4. Senoo S, Ninomiya K, Hotta K, Kiura K. Recent treatment strategy for advanced squamous cell carcinoma of the lung in Japan. Int J Clin Oncol. 2019; 24: 461-7.

5. Oberndorfer F, Mullauer L. Molecular pathology of lung cancer: current status and perspectives. Curr Opin Oncol. 2018; 30: 69-76.

6. Tao X, Yin L, Xu L, Peng J. Dioscin: A diverse acting natural compound with therapeutic potential in metabolic diseases, cancer, inflammation and infections. Pharmacol Res. 2018; 137: 259-69.

7. Zhao L, Tao X, Qi Y, Xu L, Yin L, Peng J. Protective effect of dioscin against doxorubicin-induced cardiotoxicity via adjusting microRNA-140-5p-mediated myocardial oxidative stress. Redox Biol. 2018; 16: 189-98.

8. Tao X, Xu L, Yin L, Han X, Qi Y, Xu Y, et al. Dioscin induces prostate cancer cell apoptosis through activation of estrogen receptor-beta. Cell Death Dis. 2017; 8: e2989.

9. Zhang YS, Ma YL, Thakur K, Hussain SS, Wang J, Zhang Q, et al. Molecular mechanism and inhibitory targets of dioscin in HepG2 cells. Food Chem Toxicol. 2018; 120: 143-54.

10. Z M, X H, D C, Y X, L X, L Y, et al. Potent effects of dioscin against hepatocellular carcinoma through regulating TP53-induced glycolysis and apoptosis regulator (TIGAR)-mediated apoptosis, autophagy, and DNA damage. British journal of pharmacology. 2019; 176: 919-37.

11. Wei Y, Xu Y, Han X, Qi Y, Xu L, Xu Y, et al. Anti-cancer effects of dioscin on three kinds of human lung cancer cell lines through inducing DNA damage and activating mitochondrial signal pathway. Food Chem Toxicol. 2013; 59: 118-28.

12. Lim W, Kim H, Kim Y, Choi K, Lee IH, Lee KH, et al. Dioscin suppresses TGF- $\beta 1$-induced epithelial-mesenchymal transition and suppresses A549 lung cancer migration and invasion. Bioorganic \& Medicinal Chemistry Letters. 2017; 27: 3342-8.

13. Redza-Dutordoir M, Averill-Bates DA. Activation of apoptosis signalling pathways by reactive oxygen species. Biochim Biophys Acta. 2016; 1863: 2977-92

14. Wang K, Chu D, Wu J, Zhao M, Zhang M, Li B, et al. Cinobufagin induced cell apoptosis and protective autophagy through the ROS/MAPK signaling pathway. Life Sci. 2019: 116642.

15. Wang Z, Cheng Y, Wang N, Wang DM, Li YW, Han F, et al. Dioscin induces cancer cell apoptosis through elevated oxidative stress mediated by downregulation of peroxiredoxins. Cancer Biol Ther. 2012; 13: 138-47.

16. Zhao X, Tao X, Xu L, Yin L, Qi Y, Xu Y, et al. Dioscin Induces Apoptosis in Human Cervical Carcinoma HeLa and SiHa Cells through ROS-Mediated DNA Damage and the Mitochondrial Signaling Pathway. Molecules. 2016; 21.

17. Li S, Cheng B, Hou L, Huang L, Cui Y, Xu D, et al. Dioscin inhibits colon cancer cells' growth by reactive oxygen species-mediated mitochondrial dysfunction and p38 and JNK pathways. Anticancer Drugs. 2018; 29: 234-42.

18. Cui S, Nian Q, Chen G, Wang X, Zhang J, Qiu J, et al. Ghrelin ameliorates A549 cell apoptosis caused by paraquat via p38-MAPK regulated mitochondrial apoptotic pathway. Toxicology. 2019; 426: 152267.

19. Chen B, Cao X, Lu H, Wen P, Qi X, Chen S, et al. N-(3-oxo-acyl) homoserine lactone induced germ cell apoptosis and suppressed the over-activated RAS/MAPK tumorigenesis via mitochondrial-dependent ROS in C. elegans. Apoptosis. 2018; 23: 626-40. 
20. Cao P, Xia Y, He W, Zhang T, Hong L, Zheng P, et al. Enhancement of oxaliplatin-induced colon cancer cell apoptosis by alantolactone, a natural product inducer of ROS. Int J Biol Sci. 2019; 15: 1676-84.

21. Si L, Zheng L, Xu L, Yin L, Han X, Qi Y, et al. Dioscin suppresses human laryngeal cancer cells growth via induction of cell-cycle arrest and MAPK-mediated mitochondrial-derived apoptosis and inhibition of tumor invasion. Eur J Pharmacol. 2016; 774: 105-17.

22. Guo Y, Ziesch A, Hocke S, Kampmann E, Ochs S, De Toni EN, et al. Overexpression of heat shock protein 27 (HSP27) increases gemcitabine sensitivity in pancreatic cancer cells through S-phase arrest and apoptosis. J Cell Mol Med. 2015; 19: 340-50.

23. Singh MK, Sharma B, Tiwari PK. The small heat shock protein Hsp27: Present understanding and future prospects. J Therm Biol. 2017; 69: 149-54

24. Evans I, Ko Y, Mata W, Saquib M, Eldridge I, Cohen-Gadol A, et al. Arachidonic acid induces brain endothelial cell apoptosis via p38-MAPK and intracellular calcium signaling. Microvasc Res. 2015; 98: 145-58.

25. Garrido C, Brunet M, Didelot C, Zermati Y, Schmitt E, Kroemer G. Heat shock proteins 27 and 70: anti-apoptotic proteins with tumorigenic properties. Cell Cycle. 2006; 5: 2592-601.

26. Suarez-Carmona M, Lesage J, Cataldo D, Gilles C. EMT and inflammation: inseparable actors of cancer progression. Mol Oncol. 2017; 11: 805-23.

27. Evan G, Vousden K. Proliferation, cell cycle and apoptosis in cancer. Nature. 2001; 411: 342-8.

28. Wang Y, Fu M, Liu J, Yang Y, Yu Y, Li J, et al. Inhibition of tumor metastasis by targeted daunorubicin and dioscin codelivery liposomes modified with PFV for the treatment of non-small-cell lung cancer. Int J Nanomedicine. 2019; 14: 4071-90.

29. Wang YC, Wu DW, Wu TC, Wang L, Chen CY, Lee H. Dioscin overcome TKI resistance in EGFR-mutated lung adenocarcinoma cells via down-regulation of tyrosine phosphatase SHP2 expression. Int J Biol Sci. 2018; 14: 47-56.

30. Sabharwal SS, Schumacker PT. Mitochondrial ROS in cancer: initiators, amplifiers or an Achilles' heel? Nat Rev Cancer. 2014; 14: 709-21.

31. Wu Y, Zhang J, Gao J, Li Y. Aloe-emodin (AE) nanoparticles suppresses proliferation and induces apoptosis in human lung squamous carcinoma via ROS generation in vitro and in vivo. Biochemical and Biophysical Research Communications. 2017; 490: 601-7.

32. Su L, Zhang J, Gomez H, Murugan R, Hong X, Xu D, et al. Reactive Oxygen Species-Induced Lipid Peroxidation in Apoptosis, Autophagy, and Ferroptosis. Oxidative Medicine and Cellular Longevity. 2019; 2019: 1-13.

33. Zhang Z, Rui W, Wang Z, Liu D, Du L. Anti-proliferation and anti-metastasis effect of barbaloin in non-small cell lung cancer via inactivating p38MAPK/Cdc25B/Hsp27 pathway. Oncology Reports. 2017; 38: 1172-80.

34. Ko JC, Chiu HC, Wo TY, Huang YJ, Tseng SC, Huang YC, et al. Inhibition of p38 MAPK-dependent MutS homologue-2 (MSH2) expression by metformin enhances gefitinib-induced cytotoxicity in human squamous lung cancer cells. Lung Cancer. 2013; 82: 397-406.

35. Lanneau D, Thonel AD, Maurel S, Didelot C, Garrido C. Apoptosis versus cell differentiation: role of heat shock proteins HSP90, HSP70 and HSP27. Prion. 2007; 1: 53-60.

36. Jego G, Hazoume A, Seigneuric R, Garrido C. Targeting heat shock proteins in cancer. Cancer Lett. 2013; 332: 275-85.

37. Huang ZC, Li H, Sun ZQ, Zheng J, Zhao RK, Chen J, et al. Distinct prognostic roles of HSPB1 expression in non-small cell lung cancer. Neoplasma. 2018; 65: $161-6$.

38. Fang $Y$, Wang J, Wang G, Zhou C, Wang P, Zhao S, et al. Inactivation of p38 MAPK contributes to stem cell-like properties of non-small cell lung cancer. Oncotarget. 2017; 8: 26702-17 